\title{
Features of the super prism effect in multilayer dielectric coatings
}

\author{
Yu.O. Pervak*, V.M. Onishchuk and V.Yu. Pervak \\ Taras Shevchenko Kyiv National University, Radiophysics Department; \\ e-mail: yupervak@univ.kiev.ua
}

\begin{abstract}
We demonstrate that the strong change of reflected beam intensity in the spectral range of the super-prism effect not allow to use periodic multilayer coatings as effective wavelength division multiplexing devices. But using chirped mirrors that are one of the key elements in ultrafast optics can solve this problem with success.
\end{abstract}

Keywords: chirped mirror, multilayer coating.

Manuscript received 02.09.08; accepted for publication 20.10.08; published online 11.11.08.

\section{Introduction}

The polychromatic light incident at an angle onto one of the prism surfaces is dispersed within the prism; i.e., light rays of different wavelengths propagate at different angles in the prism [1]. Splitting the light by a conventional prism relies on the dispersion of material. Due to fact that the changes in the refractive index with wavelength are rather weak for transparent materials, the achievable level of the dispersion in multilayer coatings is limited. Based on optical transparent (dielectric) materials, created were artificial structures that are known as photonic crystals [2-6]. They can be used to obtain much higher spatial dispersion. In certain conditions, photonic crystals exhibit much higher dispersion than that of the material of a conventional prism. Close to the photonic band edge, photonic crystals exhibit chromatic dispersion caused by gradual changes of the apparent refractive index, due to the curvature of the photonic bands. This can be interpreted as the prism effect, i.e. as a change in diameter of the iso-frequency lines within the band structure. If the isofrequency contours change their shape with the frequency, the dispersion can be increased by orders of magnitude. Such ultra-strong dispersive properties called super-prism effects (SPE) allow us to produce compact optical filters that are highly attractive for wavelength division multiplexing (WDM) applications [7-8]. Recently, SPE in one-dimensional thin-film structures was investigated [9-12]. Four approaches of designing the structures with a high spatial dispersion were reported. First, the strong spatial dispersion of periodic thin-film structures close to the stop-band edge was reported. Second, it was shown that the wavelengthdependent penetration depth of double chirped structures can be used to obtain spatial dispersion. Third, the coupled-cavity structures employ a wavelength dependent amount of stored energy to obtain dispersion. Fourthly, structures that use a combination of a wavelength-dependent turning point and stored energy were discussed. However, a problem of WDM intensity was not considered.

In this paper, we demonstrate that strong changes in the reflected beam intensity within the spectral range of the super-prism effect don't allow us to use periodic multilayer coatings as effective WDW devices. But using chirped mirrors can efficiently solve this problem.

\section{The periodic dielectric multilayer structures}

Let's consider the stratified infinitive media that includes infinitive repetition of two-component period LH. Here $\mathrm{L}$ and $\mathrm{H}$ are layers of different optical material with the refractive index $n_{L}$ and $n_{H}$, thickness $d_{L}$ and $d_{H}$, respectively. This stratified media is a one-dimensional photonic crystal. The optical waves with the wavelengths close to $\lambda_{0}=2\left(n_{L} d_{L}+n_{H} d_{H}\right)$ cannot spread in this media, in accordance with the theory of photonic crystals [2-6]. Generally, the full ranges of the forbidden wavelengths are obtained by methods of Bloch's waves or couple-mode theory [6]. However, the edge of a forbidden gap can be obtained by the method of equivalent layers [13]. The method of equivalent layers can be applied after a modification of the stratified infinitive media. The properties of stratified infinitive media are not changed, if we assume that media begin and finish with the layers of one eight wave optical thickness. This modification can be written as

$(\mathrm{LH})^{m} \rightarrow(0.5 \mathrm{LH} 0.5 \mathrm{~L})^{m}$, where $m \rightarrow \infty$. 
But now the stratified infinitive media can be considered as one infinitive media with equivalent refractive index of $N_{e}$, its properties and position of forbidden gap of wavelengths easy can be obtained by analysis of the characteristic matrix period

$$
\begin{aligned}
& {\left[\begin{array}{cc}
M_{11} & i M_{12} \\
i M_{21} & M_{22}
\end{array}\right]=\left[\begin{array}{cc}
\cos \delta_{L} & \frac{i}{\eta_{L}} \sin \delta_{L} \\
i \eta_{L} \sin \delta_{L} & \cos \delta_{L}
\end{array}\right] \times} \\
& \times\left[\begin{array}{cc}
\cos \delta_{H} & \frac{i}{\eta_{H}} \sin \delta_{H} \\
i \eta_{H} \sin \delta_{H} & \cos \delta_{H}
\end{array}\right]\left[\begin{array}{cc}
\cos \delta_{L} & \frac{i}{\eta_{L}} \sin \delta_{L} \\
i \eta_{L} \sin \delta_{L} & \cos \delta_{L}
\end{array}\right],
\end{aligned}
$$

where $\delta_{L}, \delta_{H}$ and $\eta_{L}, \eta_{H}$ are phase thicknesses and admittances of layers $\mathrm{L}$ and $\mathrm{H}$, respectively. Here $\delta_{L}=\frac{\pi}{\lambda} N_{L} d_{L} \cos \theta_{L} ;$ $\delta_{H}=\frac{\pi}{\lambda} N_{H} d_{H} \cos \theta_{H}$ $\eta_{r}=\chi_{\mathrm{vac}} N_{r} \cos \theta_{r}$ for TE wave and $\eta_{r}=\mathrm{X}_{\mathrm{vac}} N_{r} / \cos \theta_{r}$ for TM wave; $N_{r}=n_{r}-i k_{r}$; angles $\theta_{L}, \theta_{H}$ can be obtained from Snell's law $n_{L} \sin \theta_{L}=n_{H} \sin \theta_{H} ; r=\mathrm{L}$ or $\mathrm{H} ; \chi_{\text {vac }}$ - vacuum admittance.

Equivalent admittance is $\eta_{e}=\chi_{\mathrm{vac}} N_{e}$, where the equivalent refractive index

$$
N_{e}=\sqrt{M_{21} / M_{12}} \text {. }
$$

The equivalent refractive index is a real quantity in the case when $\left|M_{11}\right| \leq 1$. This condition defines the passband regions of the multilayer coating. In contrast, for $\left|M_{11}\right|>1$ the equivalent refractive index becomes complex and these wavelength regions are called stop bands or forbidden gaps. If stratified media is limited in such a manner that its reflectivity consist of the alternating high and low reflectivity ranges. Close to stop bands, $N_{e}$ has the high dispersion. It is the range of SPE.

Fig. 1 demonstrates reflectivity and spectral dependences of the real part of $N_{e}$ for the multilayer structure $\mathrm{S}_{0}(0.5 \mathrm{LH} 0.5 \mathrm{~L})^{m} \mathrm{~S}$, where $\mathrm{S}_{0}$-media of light incidence (air), $\mathrm{S}-$ substrate (fused silica, $n_{S} \approx 1.48$ $500 \mathrm{~nm}), \mathrm{L}$ and $\mathrm{H}$ - layers with optical thickness $\lambda_{0} / 4$ $\left(\lambda_{0}=800 \mathrm{~nm}\right)$ from silica dioxide $\left(\mathrm{SiO}_{2}\right)$ and niobium oxide $\left(\mathrm{Nb}_{2} \mathrm{O}_{5}\right), m=15-$ a number of periods. The angle of incidence is $30^{\circ}$. The reflectivity was calculated by the matrix method [13]. Reflection is

$R=\left(\frac{\eta_{0} B-C}{\eta_{0} B+C}\right)\left(\frac{\eta_{0} B-C}{\eta_{0} B+C}\right)^{*}$,

where

$$
\left(\begin{array}{l}
B \\
C
\end{array}\right)=\left[\begin{array}{cc}
M_{11} & i M_{12} \\
i M_{21} & M_{22}
\end{array}\right]^{m}\left(\begin{array}{c}
1 \\
\eta_{S}
\end{array}\right),
$$

$\eta_{0}$ and $\eta_{S}-$ admittance of air and substrate, respectively. The phase change $\varphi$ at the reflection was calculated by expression [13]

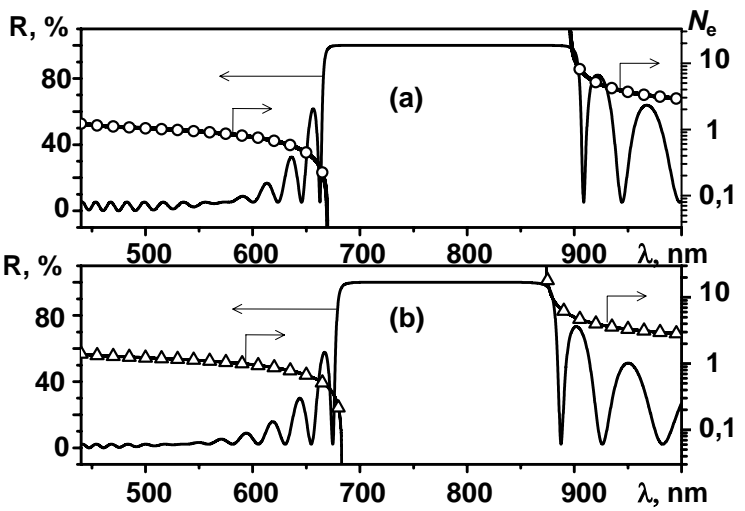

Fig. 1. Reflectivity spectra and spectral dependences of the real part of $N_{e}$ for the multilayer structure $\mathrm{S}_{0}(0.5 \mathrm{LH} 0.5 \mathrm{~L})^{15} \mathrm{~S}$ at the angle of incidence $30^{\circ}$ : (a) $s$-polarization of light; (b) $p$ polarization of light. $\mathrm{S}_{0}$-air, $\mathrm{S}-$ substrate (silica), $\mathrm{L}$ and $\mathrm{H}-$ layers with optical thickness $\lambda_{0} / 4\left(\lambda_{0}=800 \mathrm{~nm}\right)$ from silica dioxide $\left(\mathrm{SiO}_{2}\right)$ and niobium oxide $\left(\mathrm{Nb}_{2} \mathrm{O}_{5}\right)$.

$\tan \varphi=\frac{\operatorname{Im}\left[\eta_{0}\left(B C^{*}-C B^{*}\right)\right]}{\eta_{0}^{2} B B^{*}-C C^{*}}$.

The group delay (GD) is described as

$\mathrm{GD}=-\frac{d \varphi}{d \omega}=\frac{\lambda^{2}}{2 \pi c} \cdot \frac{d \varphi}{d \lambda}$,

where $c=3 \cdot 10^{8} \mathrm{~m} / \mathrm{s}$ is the speed of light.

The group delay dispersion (GDD) is

$\mathrm{GDD}=-\frac{d^{2} \varphi}{d \omega^{2}}=-\frac{\lambda^{2}}{(2 \pi)^{2}}\left(\lambda^{2} \frac{d^{2} \varphi}{d \omega^{2}}+2 \lambda \frac{d \varphi}{d \lambda}\right)$.

The material dispersion of $\mathrm{SiO}_{2}$ and $\mathrm{Nb}_{2} \mathrm{O}_{5}$ was considered for all calculations. The Cauchy formula for the refractive index of $\mathrm{SiO}_{2}$ layers is $n_{L}=1.46+$ $\left(3.35 \cdot 10^{-3} / \lambda^{2}\right)+\left(1.41 \cdot 10^{-5} / \lambda^{4}\right)$ and for $\mathrm{Nb}_{2} \mathrm{O}_{5}$ layers is $n_{H}=2.22+\left(2.18 \cdot 10^{-2} / \lambda^{2}\right)+\left(4 \cdot 10^{-3} / \lambda^{4}\right)$, if the unit of $\lambda$ is $\mu \mathrm{m}$. The stop band for TM waves ( $p$-polarization) is narrower than that for TE waves ( $s$-polarization). Note that $N_{e}$ changes from 0 to $\infty$ and it cannot be physically interpreted as a refractive index, but it is useful in analysis and synthesis of the multilayer coatings.

In the case when the angle of incidence is zero, the real part of $N_{e}$ is zero in the spectral range from 705.1 to $922.4 \mathrm{~nm}$, and the photonic forbidden gap is $217.3 \mathrm{~nm}$. When the angle of incidence equals to $30^{\circ}$, the position and width of the forbidden gap depend on light polarization. For TE wave, the width of the forbidden gap is $226.1 \mathrm{~nm}$, and it is expanded from 669.6 to $895.7 \mathrm{~nm}$. For TM wave these values are 190.1, 683 and $873.1 \mathrm{~nm}$, respectively. The longwave range to the right of the forbidden gap is the first permitted photonic band, and the shortwave range from to the left of the forbidden gap is the second permitted photonic band. The spectral 
dependences of $N_{e}$ are different and have high dispersion close to forbidden gap (Figs. 2 and 3). The multilayer structures have more periods with the shape of high reflective band close to rectangular and with higher time and spatial dispersions. The difference in the type of dispersion in the first and second permitted photonic bands causes the different dependences of the group delay in those bands. GD is the time dispersion, and it is in proportion to the spatial dispersion. The spatial shift $s$ is $[9-12]$

$$
s=v_{g x} \cdot \mathrm{GD}=\mathrm{GD} \cdot\left(\left.\frac{\partial \beta}{\partial \omega}\right|_{K=\mathrm{const}}\right)^{-1} .
$$

Here, $v_{g x}$ is a component of the group velocity along the $x$ direction, if $x$ is in the plane of incidence and parallel to layer interfaces. If $z$ is perpendicular to layer interfaces, then the wave vector $\mathbf{K}$ has components only in the $x$ and $z$ directions and is thus given by $\mathbf{K}=\beta \mathbf{x}+$ $K \mathbf{z}$ [9]. Calculations for the test structures show that the group velocity $v_{g x}$ is approximately constant with changing the wavelength, and the group delay is thus proportional to the spatial shift. This result is not completely surprising, as $v_{g x}$ is the group velocity along the layers. Within a WKB-type approximation the spatial shift and the group delay are exactly proportional, as $v_{g x}$ is independent of the wavelength [9]. This result provides physical insight and has practical consequences. The spatial and the temporal dispersion are approximately proportional; an existing structure with temporal dispersion can be modified to obtain structures with spatial dispersion. The WKB approximation (quasi-classical approximation in quantum mechanics) states that if the local wavelength $\lambda(z)$, which is linked to the local wave vector $K(z)$, changes slowly with $z$, the accumulated phase change can be calculated by integrating the wave vector $K(z)$ from the start position $z_{1}$ to the end position $z_{2}$ [9]. This result is exactly true for uniform media as well as for infinite periodic media, where the wave vector $K$ is obtained from the Bloch theory and is independent of $z$. In according to WKB approximation

$\frac{1}{v_{g x}}=\left.\left(\frac{\partial \beta}{\partial \omega}\right)_{\mathrm{appr}}\right|_{K=\mathrm{const}}=\frac{\overline{n_{\mathrm{eff}}^{2}}}{c \sin \theta}$,

where

$\overline{n_{\mathrm{eff}}^{2}}=\frac{\sum_{i}\left\{n_{i}^{2} d_{i} /\left[n_{i}^{2}-\sin ^{2} \theta\right]^{1 / 2}\right\}}{\sum_{i}\left\{d_{i} /\left[n_{i}^{2}-\sin ^{2} \theta\right]^{1 / 2}\right\}}$.

In (10) the summation is over all the layers of multilayer structure and $\theta$ is the angle of incidence, when external media is air.

For the structure $\mathrm{S}_{0}(0.5 \mathrm{LH} 0.5 \mathrm{~L})^{m} \mathrm{~S}$, where $\mathrm{S}_{0}-$ media of incidence (air), $\mathrm{S}-$ substrate (silica, $n_{S} \approx 1.48$ $500 \mathrm{~nm}$ ), L and $\mathrm{H}-$ layers with the optical thickness $\lambda_{0} / 4$
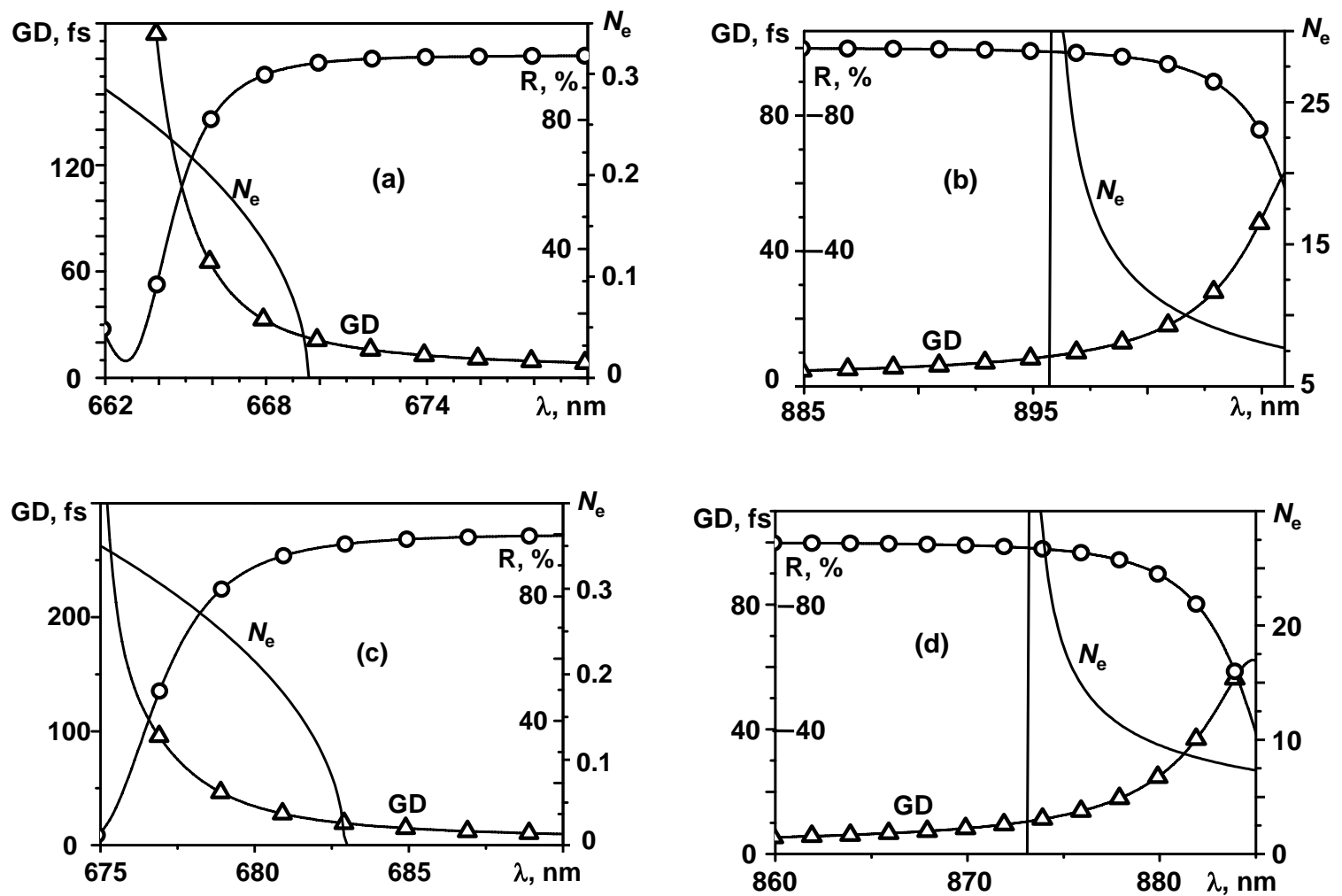

Fig. 2. Spectral dependences of $R, \mathrm{GD}$ and $N_{e}$ for the multilayer structure $\mathrm{S}_{0}(0.5 \mathrm{LH} 0.5 \mathrm{~L})^{15} \mathrm{~S}$ at the angle of incidence $30^{\circ}$. (a), (b) $s$-polarization of light; (c), (d) $p$-polarization of light. Parameters of the structure are identical to those in Fig. 1. 

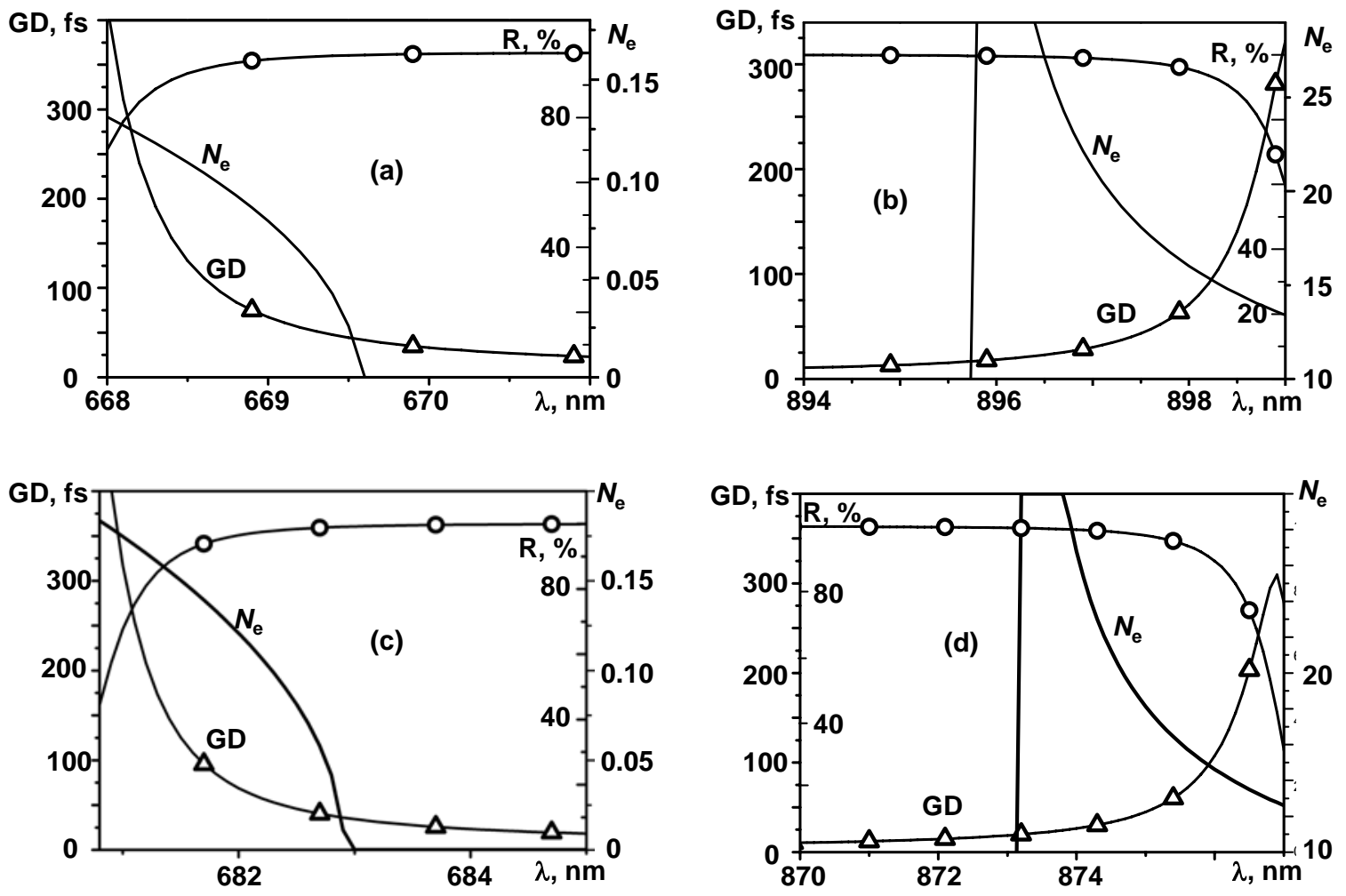

Fig. 3. Spectral dependences of $R, \mathrm{GD}$ and $N_{e}$ for multilayer structure $\mathrm{S}_{0}(0.5 \mathrm{LH} 0.5 \mathrm{~L})^{30} \mathrm{~S}$ at the angle of incidence $30^{\circ}$. (a), (b) $s$-polarization of light; (c), (d) $p$-polarization of light. Parameters of the structure are identical to those in Fig. 1.

$\left(\lambda_{0}=800 \mathrm{~nm}\right)$ from silica dioxide $\left(\mathrm{SiO}_{2}\right)$ and niobium oxide $\left(\mathrm{Nb}_{2} \mathrm{O}_{5}\right)$, it was obtained that $v_{g x}=50 \mathrm{~nm} / \mathrm{fs}$ at $\theta=30^{\circ}$. The expression (8) permits to calculate the spatial shift. Our analysis of Figs. 2 and 3 showed that the increase in layer quantity permits to increase the dispersion but decreases the spectral range with this increasing dispersion.

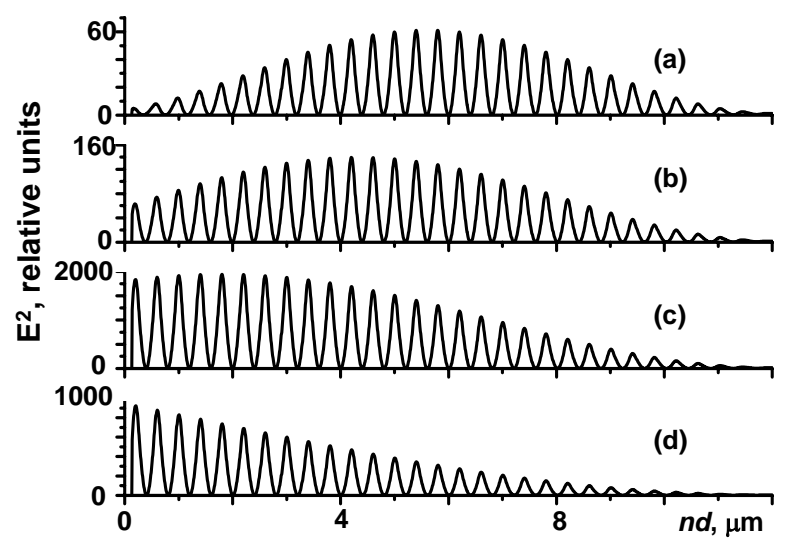

Fig. 4. Penetration of electric field through the multilayer structure of $\mathrm{S}_{0}(0.5 \mathrm{LH} 0.5 \mathrm{~L})^{30} \mathrm{~S}$ for four wavelengths: $899 \mathrm{~nm}$ (a), 898 (b), 897 (c) and 896 (d). Light is incident from the left at the angle $30^{\circ}$, and the structure extends from 0 to $12 \mu \mathrm{m}$ (optical thicknesses).
It is obvious that SPE in periodic structures is associated with the wavelength dependence of the wave penetration in the structure. In a certain sense, Fig. 4 confirms this opinion. In Fig. 4, penetration of electric field through the multilayer structure $\mathrm{S}_{0}(0.5 \mathrm{LH} 0.5 \mathrm{~L})^{30} \mathrm{~S}$ is shown for four wavelengths.

Essential imperfection of the periodic structure is a strong change of the reflectivity in the spectral range of SPE. Another imperfection is nonlinearity of the spectral dependence of GD and spatial shift. Both imperfections can be avoided, if using the chirped mirrors for demultiplexing.

\section{A chirped mirrors}

A chirped mirror is a dispersive optical interferential coating usually designed by optimizing the initial multilayer design [15-22]. A chirped mirror is characterized by a certain value of the GDD, the second derivative of the phase shift on reflection with respect to the angular frequency. A chirped mirror can provide the broadband spectrum with support, broader as for prism and grating pairs, in addition chirped mirror offers control of the third- and higher-order dispersions and higher efficiency (reflectivity) together with better beam stability. In general, the mirror GDD should compensate the material (through which the initially short pulse passes) or the (nonlinear) pulse chirps so that the 
residual dispersion fluctuations are acceptably small in all the relevant spectral range. Usually, during design optimization, unavoidable residual oscillations of GDD drop to a low level. The unavoidable GDD oscillations can broaden the pulse and lead to energy transfer from the initial single pulse to satellites. The period of the oscillations in the spectral domain determines the position of the satellite in the temporal domain, and the amplitude of these oscillations determines the amount of energy which transfered to the satellite(s).
For aims of demultiplexing, most of the problems related with chirped mirrors are not essential, and technique of chirped mirror creation is useful to obtain multilayer structures with a high dispersion. We found the nonperiodic structure that has a high dispersion and linear dependence of GD, optimization procedures are described in $[15,18]$. The structure consists of 77 alternating layers of $\mathrm{SiO}_{2}$ and $\mathrm{Nb}_{2} \mathrm{O}_{5}$ on the silica substrate. All odd layers are $\mathrm{Nb}_{2} \mathrm{O}_{5}$. The optical thicknesses of layers are shown in Fig. 5a. Most of the
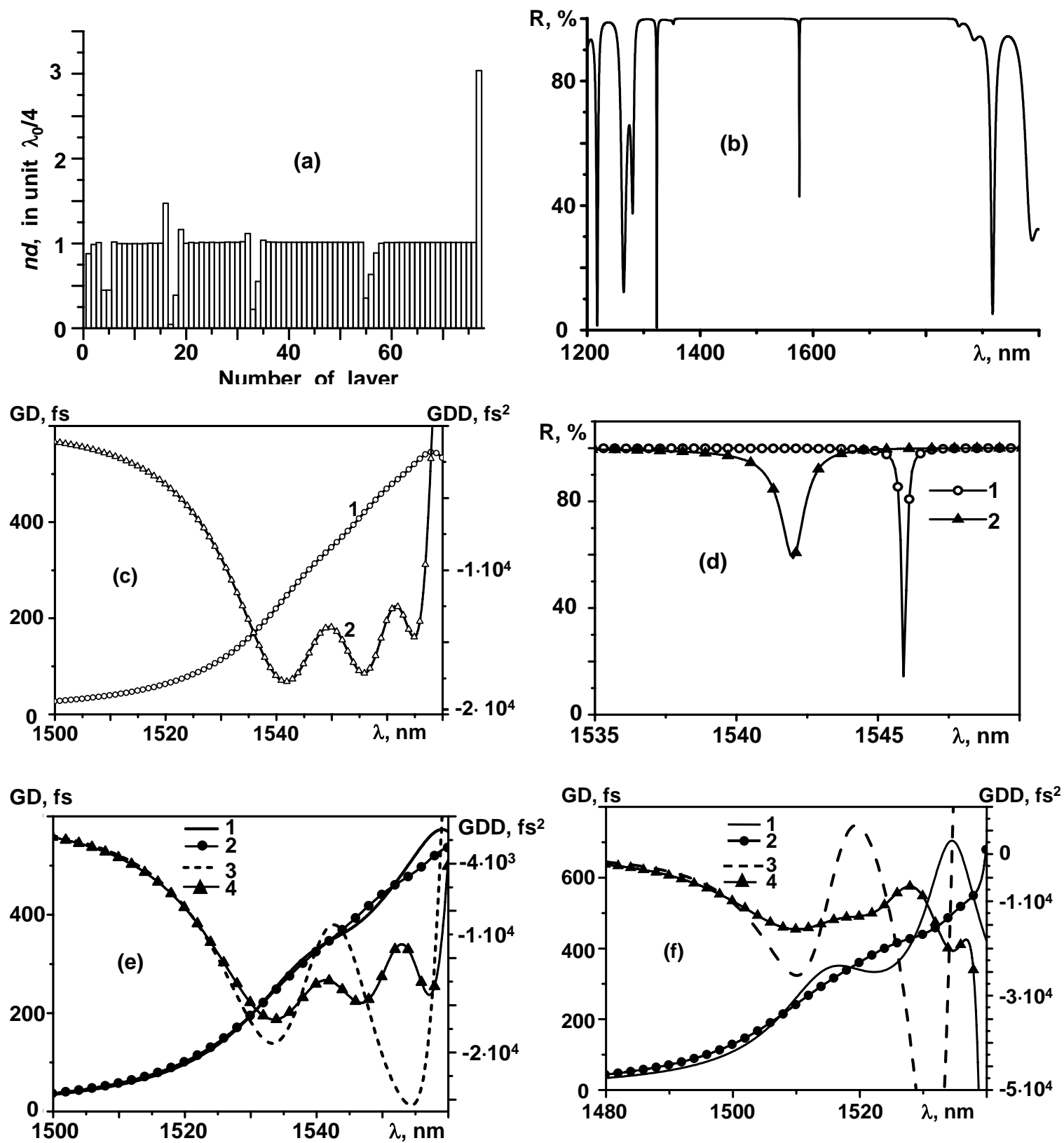

Fig. 5. Structure of the chirped mirror and spectral dependences of its reflectivity, GD and GDD at various angles of incidence: a) optical thicknesses of layers, numeration of layers begins from external media (air); b) reflectivity spectrum at normal incidence; c) spectral dependences of GD and GDD for normal incidence; d) reflectivity spectrum at the angle of incidence $20^{\circ}$ : $s$ - (1) and $p$ - (2) polarizations of light; e) spectral dependences of GD and GDD at the angle of incidence $10^{\circ}: 1-\mathrm{GD}$, $s$-polarization, 2 - GD, $p$-polarization, 3 - GDD, s-polarization, 4-GDD, p-polarization; f) spectral dependences of GD and GDD at the angle of incidence $20^{\circ}: 1-\mathrm{GD}, s$-polarization, 2-GD, $p$-polarization, 3 - GDD, s-polarization, 4-GDD, p-polarization. 
layers have the optical thickness close to $\lambda_{0} / 4\left(\lambda_{0}=\right.$ $1550 \mathrm{~nm})$, a thickness of 17-th layer is $17.6 \mathrm{~nm}, 33$ rd layer $-86.5 \mathrm{~nm}, 77$-th layer $-1177 \mathrm{~nm}$. The structure looks similar to the classical Bragg stack, it doesn't consist of the layers with a thickness close or multiple of $\lambda_{0} / 2$, and nevertheless the sharp peak of transmission is obtained in the high reflective spectral range (Figs. 5b, d). The position of this peak is $1575.7 \mathrm{~nm}$ at normal incidence, $1545.9 \mathrm{~nm}$ for $s$-polarized light at the angle of incidence $20^{\circ}$ and $1542 \mathrm{~nm}$. This important result permits to assume that the narrowband filter can be created not only on the base of Fabry-Perrot structures.

GD behaves linearly within the spectral range from 1530 to $1570 \mathrm{~nm}$ at normal incidence (Fig. 5c). Using its $\mathrm{CM}$ at oblique incidence allows to receive almost linear spectral dependence of GD in more shortwave range. Also GD behaves linearly within the spectral range from 1520 to $1560 \mathrm{~nm}$ at the angle of incidence $10^{\circ}$ independently of polarization (Fig. 5e). GD behaves linear in spectral range from 1500 to $1540 \mathrm{~nm}$ at the angle of incidence $20^{\circ}$ only for $p$-polarization (Fig. 5f). The $x$ component of the group velocity calculated using (9) and (10) is 17.37 and $34 \mathrm{~nm} / \mathrm{fs}$ for the angles of incidence $10^{\circ}$ and $20^{\circ}$, respectively. To obtain the higher group velocity, one has to use the bigger angle of incidence. The linearity of GD provides the high negative GDD within the spectral range from 1500 up to $1570 \mathrm{~nm}$.

As in the case of periodic structures, the high dispersion of chirped mirror is associated with wavelength dependence of the wave penetration in the structure. In Fig. 6, penetration of electric field through the multilayer structure of chirped mirror is shown for four wavelengths. The longwave light penetrates into the structure of chirped mirror deeper. Also, it is worth noting that the certain dispersive mirror utilizes a resonance effect, which can bring about $50 \%$ in addition to the main value of GDD obtained by the penetration depth effect [23].

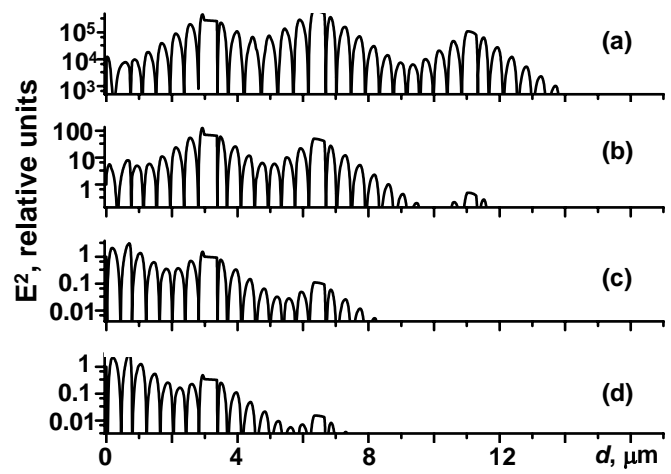

Fig. 6. Penetration of electric field through the multilayer structure of chirped mirror that is shown in Fig. 5a for four wavelengths: $1560 \mathrm{~nm}(\mathrm{a}), 1540$ (b), 1520 (c) and 1500 (d). Light is incident from the left at the angle $20^{\circ}$, and the structure extends from 0 to $16.5 \mu \mathrm{m}$ (physical thicknesses).

\section{Conclusion}

The periodic multilayer structures allow us to achieve a high dispersion within the spectral range of several nanometers. The increase in dispersion is possible when essentially increasing the number of layers. Essential drawbacks of the periodic structures demonstrate a strong change in the reflectivity and nonlinearity of the spectral dependence of GD and spatial shift in the wavelength range of SPE. Both drawbacks can be avoided by using demultiplexing the chirped mirrors.

\section{References}

1. M. Born, E. Wolf, Principles of Optics. Pergamon Press, Oxford, 1984.

2. J.D. Joannopoulos, R.D. Meade, and J.N. Winn, Photonic Crystals. Princeton University Press, Princeton, 1995.

3. S.G. Johnson, J.D. Joannopoulus, Photonic Crystals: The Road from Theory to Practice. Kluwer Academic Publ., 2002.

4. K. Sakoda, Optical Properties of Photonic Crystals. Springer, Berlin, Heidelberg, 2001.

5. K. Busch, S. Lolkes, R.B. Wehspohn, N. Foll (ed.), Photonic Crystals. Willey, New York, 2004.

6. K. Inoue, K. Ohtaka (ed.), Photonic Crystals. Springer, 2004.

7. R. Marz, S. Burger, S. Goika, A. Farchel, C. Hermann, C. Jamois, D. Michaelis, and K. Wandel, Planar high index-contrast photonic crystals for telecom applications, In: Photonic Crystals. Advances in Design, Fabrication, and Characterization. Wiley-VCH Verlag GmbH\&Co.KGaA, Weinheim, p. 308-328, 2004.

8. B. Momeni, A. Adibi, Optimization of photonic crystal demultiplexers based on the superprism effect // Appl. Phys. B 77, p. 555-560 (2003).

9. M. Gerken and D.A.B. Miller, Multilayer thin-film structures with high spatial dispersion // Appl. Opt. 42, p. 1330-1345 (2003).

10. M. Gerken and D.A.B. Miller, Wavelength demultiplexer using the spatial dispersion of multilayer thin-film structures // IEEE Photon. Technol. Lett. 15, p. 1097-1099 (2003).

11. M. Gerken, Wavelength multiplexing by spatial beam shifting in multilayer thin-film structures // Electrical Engineering Ph.D. Dissertation, Stanford Univ., Stanford, Calif., 2003.

12. M. Gerken, D.A.B. Miller, Limits on the performance of dispersive thin-film stacks // Appl. Opt. 44, No.16, p. 3349-3357 (2005).

13. H.A. MacLeod, Thin-Film Optical Filters. Institute of Phys. Publish., Philadelphia, Pa., 2001.

14. N. Matuschek, F.X. Kärtner, and U. Keller, Theory of doublechirped mirrors // IEEE J. Sel. Top. Quantum Electron. 4, p. 197-208 (1998).

15. A. Fernandez, A. Verhoef, V. Pervak, G. Lermann, F. Krausz, A. Apolonski, Generation of $60-n J$ sub- 
40-fs pulses at $70 \mathrm{MHz}$ repetition rate from a Ti:sapphire chirped pulse-oscillator // Appl. Phys. B 87, p. 395-398 (2007).

16. R. Szipöcs, K. Ferencz, C. Spielmann, and F. Krausz, Chirped multilayer coatings for broadband dispersion control in femtosecond lasers // Opt. Lett. 19, p. 201-203 (1994).

17. V. Pervak, S. Naumov, G. Tempea, V. Yakovlev, F. Krausz, A. Apolonski, Synthesis and manufacturing the mirrors for ultrafast optics // Proc. SPIE 5963, p. 490-499 (2005).

18. V. Pervak, A.V. Tikhonravov, M.K. Trubetskov, S. Naumov, F. Krausz, A. Apolonski, 1.5-octave chirped mirror for pulse compression down to sub3 fs // Appl. Phys. B 87, p. 5-12 (2007).

19. G. Steinmeyer, G. Stibenz, Generation of sub-4-fs pulses via compression of a white-light continuum using only chirped mirrors // Appl. Phys. B 82, p. 175-181 (2006).
20. G. Steinmeyer, Femtosecond dispersion compensation with multiplayer coatings: toward the optical octave // Appl. Opt. 45, p. 1484-1490 (2006).

21. N. Matuschek, L. Gallmann, D.H. Sutter, G. Steinmeyer, U. Keller, Back-side-coated chirped mirrors with ultra-smooth broadband dispersion characteristics // Appl. Phys. B 71, p. 509-522 (2000).

22. F.X. Kärtner, N. Matuschek, T. Schibli, U. Keller, H. A. Haus, C. Heine, R. Morf, V. Scheuer, M. Tilsch, and T. Tschudi, Design and fabrication of double-chirped mirrors // Opt. Lett. 22, p. 831-833 (1997).

23. V. Pervak, C. Teisser, A. Sugita, S. Naumov, F. Krasz, A. Apolonski, High-dispersive mirrors for femtosecond lasers // Optics Express 16, No.14, p. 10220-10233 (2008). 